\title{
A model established using marital status and other factors from the Surveillance, Epidemiology, and End Results database for early-stage gastric cancer
}

\section{Lixiang Zhang}

Department of General Surgery, The First Affiliated Hospital of Anhui Medical University, Hefei, 230031, China

\section{Baichuan Zhou}

Department of General Surgery, The First Affiliated Hospital of Anhui Medical University, Hefei, 230031, China

\section{Panquan Luo}

Department of General Surgery, The First Affiliated Hospital of Anhui Medical University, Hefei, 230031, China

\section{Aman Xu (D903319323@qq.com)}

First Affiliated Hospital of Anhui Medical University https://orcid.org/0000-0001-8142-4478

\section{Wenxiu Han}

Department of General Surgery, The First Affiliated Hospital of Anhui Medical University, Hefei, 230031, China

\section{Zhijian Wei}

Department of General Surgery, The First Affiliated Hospital of Anhui Medical University, Hefei, 230031, China

\section{Research}

Keywords: early-stage gastric cancer, marital status, surgery, prognosis, cancer

Posted Date: March 4th, 2021

DOl: https://doi.org/10.21203/rs.3.rs-274394/v1

License: (9) This work is licensed under a Creative Commons Attribution 4.0 International License. Read Full License 


\section{Abstract \\ Background}

Currently, the postoperative prognosis of early-stage gastric cancer (GC) is difficult to accurately predict. Therefore, this study aimed to combine the basic data, clinical indicators, and treatment information of patients to establish a predictive model for early-stage GC based on a new scoring system.

\section{Methods}

A total of 3647 patients with early stage GC from the Surveillance, Epidemiology, and End Results (SEER) database were included in this study. A Kaplan-Meier survival analysis was used to compare differences in prognosis between different marital status, as an innovative prognostic indicator. Univariate and multivariate analyses were used to screen available prediction factors and then build a nomogram using the Cox proportional hazard regression model. Calibration curves and C-index were used to verify the prediction model.

\section{Results}

The univariate analysis showed that age at diagnosis, sex, histology, stage_T and N GCs, surgery in the primary site, lymph node dissection, chemotherapy, radiation, tumor size, and marital status were significant prognostic factors of GC. The multivariate analysis revealed that age at diagnosis, sex, histology, stage_T, surgery, tumor size, and marital status were independent prognostic factors of overall survival. Both the $\mathrm{C}$-index and calibration curves confirmed that the nomogram had a great predictive effect on patient prognosis in training and testing sets.

\section{Conclusion}

We established an accurate prediction model for the postoperative prognosis of patients with early-stage GC based on various clinical indicators and treatment information. This nomogram can effectively help patients with early-stage GC in the future.

\section{Introduction}

Gastric cancer (GC) is the fifth most common cancer and third leading cause of cancer-related deaths globally, with over 1 million new cases of GC and about 780000 deaths in 2018 [1] [2] [3]. In the past few decades, GC has been a main factor that has increased disability-adjusted life years globally, especially in areas with a GC high incidence, such as Japan, China, and other Asian regions [4] [5]. GC is approximately two- to three-folds higher in men than in women and is uniformly rare in young people aged $<50$ years [6], with increasing incidence rates after 50 years age. Early-stage GC is defined as GC limited in the lamina 
propria, mucosa, or submucosa, regardless of lymph node metastasis. Early-stage GC has a greater chance of successfully getting removed through radical resection than advanced GC, consequently having a better prognosis than that of the latter. Therefore, it is essential to diagnose and treat GC early to improve prognosis.

Even for patients with early-stage GC who underwent systematic treatment, accurately predicting GC prognosis is difficult. Therefore, it is meaningful to establish a reliable predictive model in combination with post-treatment indicators. We obtained a large amount of clinical data regarding patients with earlystage GC from the Surveillance, Epidemiology, and End Results (SEER) database to acquire a

large sample size and great authenticity and incorporate various research indicators. According to current research, tumor size, stage_N, histology, age at diagnosis, tumor location, and other factors can affect survival [2] [7] [8]. However, these indicators have limited clinical developments, such as the wide application of endoscopic surgery (endoscopic mucosal resection [EMR] and endoscopic submucosal dissection [ESD]). These organ-sparing therapies remain problematic for cancer cells and metastatic lymph node residues [9] [10]; they also make fast recovery [11] [12]. Therefore, early GC treatment may also be a prognostic factor for cancer patients. Moreover, there are also other indicators that are related to the prognosis of cancer patients. Marital status, which is associated with prostate, cervical, and rectal cancer [13] [14], has emerged as an innovative risk factor in recent years. Some reports studied the impact of race on survival [15] [16]; these reports studied multiple races and were different from traditional studies that had only focused on one race. Chemotherapy has been proven to be effective for treating GC in long-term clinical applications. In recent years, neoadjuvant chemotherapy has become an important part of the treatment of advanced GC [17], but the effect of chemotherapy on early-stage GC remains controversial.

To the best of our knowledge, few studies have focused on the effect of these early-GC indicators. Therefore, we performed a nomogram that can assess the impact of various indexes comprehensively to provide a basis for the prediction of the overall survival (OS) of early-stage GC patients.

\section{Patients And Methods}

\section{Data Source and Patient Selection}

In this study, we acquired data from the SEER database of patients with gastric cancer to evaluate the degree of the aforementioned factors. A nomogram, which is stable and visible, was used in our data analysis. A nomogram is based on multivariate analyses and integrates multiple predictive indicators; it can be used to diagnose diseases and predict their incidence or progression. We built a prediction nomogram based on independent accurate GC predictors. This gave us the ability to select an optional therapeutic regimen for individual patients. Research was restricted to tumors limited to the lamina propria, mucosa, and submucosa. Exclusion criteria in our study were as follows: (a). benign or stromal tumors; (b). distant metastasis or distant lymph node metastases; (c). second malignant primary indicator; (d). unknown chemotherapy; and (e). unknown survival time. Finally, 3647 cases were screened 
and included in this study. They were randomly divided into two groups-training and testing sets-based on a 3:1 ratio, respectively, meaning that 2719 people were in the training set and 928 people were in the testing set.

\section{Data Collection and End Point}

The following variables were included in our study: age at diagnosis, race, gender, tumor location, histology, grade, stage_T and N GCs, surgery in the primary site, lymph node dissection, chemotherapy, radiation, tumor size, insurance, and marital status. The main end point was OS, which was defined as the time from diagnosis until death due to any reason.

Age was divided into seven subgroups: $\leq 40,40-50,50-60,60-70,70-80,80-90$, and 90-100 years; race was divided into three subgroups: white, black, and other; the "other" subgroup included Indian, Asian, and other minorities; tumor site was classified into eight subgroups according to the anatomy of the stomach: fundus, body, antrum, pylorus, lesser curvature, greater curvature, gastric overlapping area, and NOS; based on ICD-O-3 (International Classification of Disease for Oncology), histology was divided into five subgroups: adenocarcinoma, signet ring cell carcinoma, special-type carcinoma, including carcinoid tumor, goblet cell carcinoid, and squamous cell carcinoma, other carcinomas, including neoplasms, diffuse type carcinoma, and linitis plastica, and unknown. Surgery was divided into five subgroups: no cancer-direct surgery, endoscopic surgery, partial gastrectomy, total gastrectomy, and unknown; lymph node dissection was divided into four subgroups: none, 1-3 regional lymph nodes removed, 4 or more regional lymph nodes removed, and unknown; and tumor size was divided into eight subgroups: invisible to the naked eyes, $\leq 1 \mathrm{~cm}, 1-2 \mathrm{~cm}, 2-3 \mathrm{~cm}, 3-4 \mathrm{~cm}, 4-5 \mathrm{~cm},>5 \mathrm{~cm}$ or widespread, and unknown. Marital status was divided into six subgroups: married (including domestic partner), divorced, separated, widowed, single (never married), and unknown.

\section{Statistical Methods}

Continuous variables were expressed as mean \pm standard deviations. Categorical variables were identified by frequency and proportion, which were both analyzed by Student's t-test and Pearson's chisquare and Fisher's exact tests. A Kaplan-Meier analysis was performed to describe and compare survival among different variables, and parameters included mean and median survival times with a 95\% confidence interval $(\mathrm{Cl})$. We also performed the log-rank test to compare the significance of survival curves. In the Cox proportional hazards regression analysis, variables that were considered significant in the univariate analysis were put in the multivariate analysis. These indicators that were ultimately meaningful were used to establish a nomogram to predict 3- and 5-year OSs. The parameters of the Cox proportional hazards regression analysis included hazard ratios and 95\% Cls. The C-index was employed to measure the reliability of the nomogram. We also built calibration curves to examine outcomes. All data were analyzed using SPSS (version 23.0) and R software (version 3.4.3).

\section{Results}




\section{Baseline Characteristics}

A total of 141954 patients were extracted from the SEER database, and 3647 suitable patients with earlystage GC were included in this study. We divided patients into two cohorts-training ( $n=2719,75 \%$ of data) and testing sets ( $n=928,25 \%$ of data). Of the included patients, 1793 (49.2\%) were male and 1854 (50.8\%) were female. Moreover, 2231 were white, 607 were black, and 809 were put in the "other" race subgroup. Regarding marital status, 1957 were married, 274 were divorced, 41 were separated, 630 were widowed, 512 were single, and 233 were classified into the "unknown" group. Baseline characteristics of the training set is shown in Table 1. Age at diagnosis $(P<0.001)$, race $(P<0.001)$, gender $(P<0.001)$, histology $(P=0.007)$, grade $(P=0.009)$, stage_T $(P=0.025)$ and $N(P<0.001) G C s$, surgery $(P<0.001)$, tumor size $(P=0.005)$, and insurance $(P<0.001)$ were significantly different among marital status groups.

\section{TABLE 1}

\section{Kaplan-Meier Survival Analysis of Marital Status Groups}

To explore the influence of different marital status groups on OS, the Kaplan-Meier survival analysis was performed in all patients in the training set. As shown in Figure 1, married individuals had the best prognosis (average OS=72.084, 95\% $\mathrm{Cl}=70.847-73.321$ ), and the OS of widows was the worst (average $\mathrm{OS}=60.150,95 \% \mathrm{Cl}=57.057-63.244)$. To verify whether gender is related to the abovementioned results, we conducted the Kaplan-Meier survival analysis in GC patients of different genders. As shown in Figure $2 A-B$, there were significant differences in $0 S$ between sexes $(P<0.001)$. In both male and female patients with early-stage GC, survival was highest for married individuals (male average, OS $=69.187,95 \%$ $\mathrm{Cl}=67.446-70.928$; and female average, $\mathrm{OS}=76.357,95 \% \mathrm{Cl}=74.783-77.930)$, and survival was the worst in widows (male average, OS $=51.704,95 \% \mathrm{Cl}=45.206-58.202$; and female average $\mathrm{OS}=61.885,95 \%$ $\mathrm{Cl}=58.476-65.293)$. It is worthy to note that survival was significantly better in divorced female patients than in divorced male patients. Simultaneously, we also performed the Kaplan-Meier analysis of each known marital status group among genders, except for the "separated" group as it had a small sample size and was consequently of limited reference. As shown in Figure 3 , there were significant differences between male and female patients in each marital status group (married, $P<0.001$, Figure $3 \mathrm{~A}$; divorced, $P=0.020$, Figure 3B; widowed, $P=0.025$, Figure $3 C$; and single, $P=0.026$, Figure $3 D$ ). Survival was better in female patients than in male patients.

\section{Prognostic Factors of GC Patients}

Univariate analysis results are shown in Table 1. The analysis showed that age at diagnosis, gender, histology, stage_T and N GCs, surgery, lymph node dissection, chemotherapy, radiation, tumor size, and marital status were significant prognostic factors. These univariate analysis factors were included in the multivariate analysis. Multivariate results showed that age at diagnosis, sex, histology, stage_T, surgery, tumor size, and marital status were independent prognostic factors for OS (Table 2).

\section{Prognostic Nomogram for OS}


Based on Cox regression models, a nomogram was constructed to predict the 3 - and 5-year OSs of patients with early-stage GC (Figure 4). This nomogram created a scoring system in which each included variable can obtain a corresponding score of 0 to 100 according to their contribution to OS. After these scores were added to calculate the total score, the corresponding OS was predicted based on the scale at the bottom of the figure. This nomogram showed that tumor size was the most important prognosis factor, followed by age at diagnosis and surgery. Stage_T, marital status, gender, and histology also have a moderate impact on the prognosis of patients with early-stage GC. The nomogram obtained in our study had good predictive ability and reliability.

\section{Validation of the Nomogram}

In this study, we built a model that can predict the prognosis of patients with GC based on the SEER database; this model was validated by the testing set. The $C$ index was 0.791 and 0.685 in the training and testing sets, respectively, demonstrating that our nomogram was useful for patients with GC. Simultaneously, a calibration curve was used to examine the nomogram's ability to predict the 3- and 5year OSs of patients of training and testing sets. As shown in Figure 5, the prediction of the nomogram was closely related to the observed results. We also performed an receiver operating characteristic curve; the 3-year survival area under the curve (AUC) was 0.774 and 0.717 in training and testing sets respectively, and the 5-year AUC was 0.773 and 0.722, as shown in Figure 6.

\section{Kaplan-Meier Curves for Nomogram}

According to scoring results, we divided patients into high-risk and low-risk groups (high-risk group and low-risk group were bounded by the median of the risk score) and performed the Kaplan-Meier survival analysis on these groups. As shown in Figure 6, there was a significant difference between the KaplanMeier curves of the high-risk group and those of the low-risk group, further demonstrating the reliability of the nomogram.

\section{Discussion}

GC has two of the highest morbidity and fatality rates among cancers, originating from the gastric mucosal epithelium [2]. It can grow in various sites of the stomach and can easily develop hematogenous or lymphatic metastases [3]. In recent years, GC started to occur in young patients [1]. It is known that, even at early stages, GC may recur or develop metastases; therefore, it is important to maintain routine treatment and reviews to prolong patient survival [18]. Every coin has two sides; excessive treatment and examination will increase the financial burden on patients; however, it will affect GC prognosis. For example, enhanced computed tomography, which is effective in diagnosing GC, is not only expensive but also extremely unhealthy. Therefore, it is important to build a reliable nomogram that can accurately evaluate the recurrence risk of patients with GC postoperatively. Many studies revealed few GC prognostic factors, such as tumor size and invasion depth. However, these factors were limited and focused only on tumor growth and not on the patients' general condition and treatment information. Our research was based on the SEER database and included different races, innovatively adding some indicators that were 
proven to be associated with many kinds of cancer [13] [14]; such indicators, such as marital status, are rarely used for GC. Although some studies have used nomograms to predict the prognosis of patients with GC [19] [20] [21], we attempted to establish a prognostic nomogram combining multifarious clinical indicators, pathological characteristics, and treatment information to evaluate the probability of 3-and 5year OSs of such patients.

In our study, the nomogram was more credible and persuasive as the outcomes were obtained from the data of the training set and then validated by testing set. First, we performed a univariate analysis including all factors; of these factors, we selected those that were significant, including age, sex, histology, and surgery, and brought them into the multivariate analysis. The multivariate analysis revealed that age at diagnosis, sex, histology, stage_T, surgery, tumor size, and marital status were independent prognostic factors of OS. A nomogram was constructed based on these factors, and the Cindex was 0.791 . Calibration curves showed great consistency between prediction and observation results, and there was a significant difference between the high-risk and low-risk groups. Moreover, the AUCs of 3- and 5-year survivals were 0.774 and 0.773 , respectively.

The nomogram has been continuously proven to be a reliable and accurate prognostic prediction tool in recent studies. It can evaluate survival using various comprehensive indicators and acquire a better prediction effect than other prediction tools.

For patients with early-stage GC, based on the nomogram obtained in this study, combined with clinical information, we can obtain a postoperative patient risk rating.

For high-risk patients, review frequency and follow-up times should be increased.

Patients themselves should pay more attention to symptom fluctuation and improvements in lifestyle.

From the seven factors included in our nomogram, tumor size was the largest contributor to OS. This is in line with our usual perception, which is that a larger tumor is more aggressive and that a barely visible carcinoma in situ is indolent. Ohashi et al. thought that both tumor size and depth could be used as combined prognostic indicators [22]. Our scoring system also included tumor invasion depth, and the T1b score was moderately higher than the T1a score. Tumors with a higher T stage have deeper infiltration, and there are more vascular and lymphatic vessels in the submucosa than in the mucosa, causing tumor cells to spread further and making them more difficult to remove; this directly worsens patient survival. Age was also an OS risk factor. Looking at the overall trend, old patients scored higher nomogram scores and had a worse prognosis than young patients.

This might be attributed to the fact that elderly patients have a worse general condition and immune tumor cell clearance and more underlying diseases than the former.

It is worthy to note that different surgical methods also have a certain impact on prognosis. Patients who did not undergo surgery had the worst prognosis, indicating that surgery is still the most effective treatment for GC. Patients who underwent partial gastrectomy scored best on the nomogram, while patients who underwent endoscopic surgery and total gastrectomy had similar scores. This does not 
mean that total gastrectomy is not effective for treating GC because the condition of patients who need total gastrectomy might be more serious. Whether partial gastrectomy or endoscopic surgery is better for early-stage small-diameter GC has remained a controversial issue in clinical practice, and a few studies have been dedicated to provide references to choose the correct treatment. Nishizawa et al. indicated that patients receiving ESD generally had a better quality of life postoperatively; however, they also had a higher incidence of metachronous GC [23]. Mun et al. reported that endoscopic surgery has fewer complications than traditional surgery based on the fact that the OS of the former is no less than that of the latter [11]. Nomura et al. suggested that we should try to reduce the extent of gastrectomy if GC can still be cured [24]. Our nomogram showed that partial gastrectomy was generally better than endoscopic surgery, indicating that, according to the current medical level, we should be cautious in using endoscopic surgery instead of traditional surgery. For patients with large tumor diameters and poor histological types, partial gastrectomy should be preferred to ensure radical resection. Because of its lower trauma rate and higher safety, endoscopic surgery can be applied to patients who cannot tolerate surgery due to advanced age or underlying diseases.

Marital status, a factor that is rarely enrolled in GC research, also showed moderate influence on survival in our nomogram.

Married patients had the best prognosis, followed by single patients, and the prognosis of separated patients was the worst.

This result was consistent with the Kaplan-Meier survival curve. We speculate that this might be due to the fact that married patients had better financial conditions and emotional encouragement, while separated patients might be more likely to experience financial difficulties emotional loss. Moreover, our nomogram showed that gender and histology also had an important impact on prognosis. Male patients had higher scores and poorer prognoses. This might be related to genetic differences between men and women. Li et al. reported that the expression of different core genes and differences in pathways were associated with the variation observed among patients with GC of different races and sexes [25].

Different lifestyles as a result of different sexes might also affect prognosis.

Considering histology, signet ring cell carcinoma had the worst survival according to the nomogram.

This result is also consistent with traditional clinical knowledge. Riihimäki et al. demonstrated that signet ring adenocarcinomas had a higher probability of metastasis within the peritoneum, bone, and ovaries than adenocarcinomas [26]. The higher risk of metastasis may be the reason for the worse prognosis in patients with signet ring cell carcinoma.

To the best of our knowledge, this is the first SEER-based nomogram combining comprehensive clinical indicators to predict OS in patients with early-stage GC. However, our research has some limitations.

To improve the reliability of our study, we divided the screened SEER data into training and testing sets at a ratio of 3:1; however, the validation of the local medical center data was still missing.

Second, retaining unclearly classified data or data displayed as "unknown" not only enlarged the scope of application of the nomogram, but also increased mutual interference between data to a certain extent; 
this affected the accuracy of the nomogram. Third, some well-known risk factors of GC, such as family history, alcohol and Helicobacter pylori (HP) infection, were not enrolled. These indicators were scarce in the SEER database as it was difficult to acquire them. For example, there is no clear standard to determine whether the patient has a history of drinking alcohol based on the amount of alcohol consumed, the frequency of drinking, and the time of abstinence. Moreover, HP infection examinations are not routinely performed in many areas, making it difficult for the data to be applied in large databases.

\section{Conclusion}

In conclusion, our nomogram included age at diagnosis, sex, T stage, histology, tumor size, surgery, and marital status as risk factors and effectively predicted the prognosis of early-stage GC. This nomogram can help assess the prognosis and treatment of patients with GC.

\section{Declarations}

\section{Ethics statement}

Our study was reviewed and approved by the Ethics Committee of the First Affiliated Hospital of Anhui Medical University. The data involved in the research were all available on the Internet.

\section{Consent of publication}

Written informed consent was obtained from the authors for the publication of any potentially indentifiable images or data included in our manuscript.

\section{Data availability statement}

Publicly available datasets were analyzed in this study. This data can be found here: http://seer.cancer.gov/data/.

\section{Competing interests}

The authors declare that the research was conducted in the absence of any commercial or financial relationships that could be construed as a potential conflict of interest.Funding

This research did not accept financial support from any organization or individual.

\section{Authors' contributions}

Li-xiang Zhang and Bai-chuan Zhou made the contribution to the main work equally, they designed the study. Li-xiang Zhang provided the databases. Pan-quan Luo, A-man Xu, Wen-xiu Han and Zhi jian Wei provided help and analyzed the data. Bai-chuan Zhou wrote the manuscript. All authors contributed to the article and approved the submitted version. 


\section{Acknowledgements}

Not applicable

\section{Authors' information}

All authors come from department of General Surgery, The First Affiliated Hospital of Anhui Medical University, Hefei, China

\section{References}

1. Bray F. Ferlay J, Soerjomataram I. Siegel RL, Torre LA. Jemal A. Global cancer statistics 2018 : GLOBOCAN estimates of incidence and mortality worldwide for 36 cancers in 185 countries. CA Cancer J Clin. 2018 Nov;68(6):394-424. doi: 10.3322/caac.21492. Epub 2018 Sep 12. Erratum in: CA Cancer J Clin. 2020 Jul;70(4):313. PMID: $30207593 .$.

2. Venerito M. Link A, Rokkas T. Malfertheiner P. Gastric cancer - clinical and epidemiological aspects. Helicobacter. 2016 Sep;21 Suppl 1:39-44. doi: 10.1111/hel.12339. PMID: 27531538.

3. Smyth EC. Nilsson M, Grabsch HI. van Grieken NC, Lordick F. Gastric cancer. Lancet. 2020 Aug 29;396(10251):635-648. doi: 10.1016/S0140-6736(20)31288-5. PMID: 32861308..

4. Lin JT. Screening of gastric cancer: who, when, and how. Clin Gastroenterol Hepatol. 2014 Jan;12(1):135-8. doi: 10.1016/j.cgh.2013.09.064. Epub 2013 Oct 6. PMID: $24107396 .$.

5. Kobayashi M. Sato Y, Terai S. Endoscopic surveillance of gastric cancers after Helicobacter pylori eradication. World J Gastroenterol. 2015 Oct 7;21(37):10553-62. doi: 10.3748/wjg.v21.i37.10553. PMID: 26457015; PMCID: PMC4588077..

6. Recio-Boiles A. Babiker HM. Gastric Cancer. 2020 Dec 9. In: StatPearls [Internet]. Treasure Island (FL): StatPearls Publishing; 2020 Jan-. PMID: 29083746.

7. Bilici A. Treatment options in patients with metastatic gastric cancer: current status and future perspectives. World J Gastroenterol. 2014 Apr 14;20(14):3905-15. doi: 10.3748/wjg.v20.i14.3905. PMID: 24744580; PMCID: PMC3983446..

8. Liang Y. Wu L, Liu L. Ding X, Wang X. Liu H, Meng J. Xu R, He D. Liang H. Impact of extranodal tumor deposits on prognosis and N stage in gastric cancer. Surgery. 2019 Sep,166(3):305-313. doi: 10.1016/j.surg.2019.04.027. Epub 2019 Jun 18. PMID: 31221435.

9. Park SE. Kim SH, Kim SG. Han JK. Local or extragastric recurrence after incomplete endoscopic submucosal dissection of early gastric cancer: risk factors and the role of CT. Abdom Radiol (NY). 2018 Dec;43(12):3250-3259. doi: 10.1007/s00261-018-1659-0. PMID: $29948060 .$.

10. Liu Q. Ding L, Qiu X. Meng F. Updated evaluation of endoscopic submucosal dissection versus surgery for early gastric cancer: A systematic review and meta-analysis. Int J Surg. 2020 Jan;73:2841. doi: 10.1016/j.ijsu.2019.11.027. Epub 2019 Nov 26. PMID: 31783166. 
11. Venerito M. Link A, Rokkas T. Malfertheiner P. Gastric cancer- clinical and epidemiological aspects. Helicobacter. 2016 Sep;21 Suppl 1:39-44. doi: 10.1111/hel.12339. PMID: 27531538.

12. Mun YG. Choi MG, Lim CH. Lee HH, Kang DH. Park JM, Song KY. Factors Affecting Endoscopic Curative Resection of Gastric Cancer in the Population-Based Screening Era. Clin Endosc. 2018 Sep;51(5):478-84. doi: 10.5946/ce.2018.006. Epub 2018 Jun 1. PMID: 29852731; PMCID: PMC6182285.

13. Ono H. Yao K, Fujishiro M. Oda I, Nimura S. Yahagi N, lishi H. Oka M, Ajioka Y. Ichinose M, Matsui T. Guidelines for endoscopic submucosal dissection and endoscopic mucosal resection for early gastric cancer. Dig Endosc 2016 Jan,28(1):3-15. doi: 10.1111/den.12518. Epub 2015 Oct 4. PMID: 26234303.

14. Khan S. Nepple KG, Kibel AS. Sandhu G, Kallogjeri D. Strope S, Grubb R 3rd. Wolin KY, Sutcliffe S. The association of marital status and mortality among men with early-stage prostate cancer treated with radical prostatectomy: insight into post-prostatectomy survival strategies. Cancer Causes Control. 2019 Aug,30(8):871-876. doi: 10.1007/s10552-019-01194-y. Epub 2019 Jun 18. PMID: 31214808; PMCID: PMC6739072.

15. Machida H. Eckhardt SE, Castaneda AV. Blake EA, Pham HQ. Roman LD, Matsuo K. Single Marital Status and Infectious Mortality in Women With Cervical Cancer in the United States. Int J Gynecol Cancer. 2017 Oct,27(8):1737-1746. doi: 10.1097/IGC.0000000000001068. PMID: 28704326; PMCID: PMC7526045.

16. Diao JD. Wu CJ, Cui HX. Bu MW, Yue D. Wang X, Liu YL. Yang YJ. Nomogram predicting overall survival of rectal squamous cell carcinomas patients based on the SEER database: A populationbased STROBE cohort study. Medicine (Baltimore). 2019 Nov,98(46):e17916. doi: 10.1097/MD.0000000000017916. PMID: 31725640; PMCID: PMC6867783.

17. Ellis L. Canchola AJ, Spiegel D. Ladabaum U, Haile R. Gomez SL. Racial and Ethnic Disparities in Cancer Survival: The Contribution of Tumor, Sociodemographic, Institutional, and Neighborhood Characteristics. J Clin Oncol. 2018 Jan 1,36(1):25-33. doi: 10.1200/JC0.2017.74.2049. Epub 2017 Oct 16. PMID: 29035642; PMCID: PMC5756323.

18. Saumoy M. Schneider Y, Shen N. Kahaleh M, Sharaiha RZ. Shah SC. Cost Effectiveness of Gastric Cancer Screening According to Race and Ethnicity. Gastroenterology 2018 Sep;155(3):648-60. doi: 10.1053/j.gastro.2018.05.026. Epub 2018 May 17. PMID: 29778607.

19. Coccolini F. Nardi M, Montori G. Ceresoli M, Celotti A. Cascinu S, Fugazzola P. Tomasoni M, Glehen O. Catena F, Yonemura Y. Ansaloni L. Neoadjuvant chemotherapy in advanced gastric and esophagogastric cancer. Meta-analysis of randomized trials. Int J Surg. 2018 Mar;51:120-7. doi: 10.1016/j.ijsu.2018.01.008. Epub 2018 Feb 20. PMID: 29413875.

20. Findlay JM, Antonowicz S, Segaran A, El Kafsi J, Zhang A, Bradley KM, Gillies RS, Maynard ND, Middleton MR. Routinely staging gastric cancer with 18F-FDG PET-CT detects additional metastases and predicts early recurrence and death after surgery. Eur Radiol. 2019 May,29(5):2490-2498. doi: 10.1007/s00330-018-5904-2. Epub 2019 Jan 14. PMID: 30643947; PMCID: PMC6443603. 
21. Yu C. Zhang Y. Development and validation of prognostic nomogram for young patients with gastric cancer. Ann Transl Med. 2019 Nov;7(22):641. doi: 10.21037/atm.2019.10.77. PMID: 31930042; PMCID: PMC6944578.

22. Bando E. Ji X, Kattan MW. Seo HS, Song KY. Park CH, Bencivenga M. de Manzoni G, Terashima M. Development and validation of a pretreatment nomogram to predict overall survival in gastric cancer. Cancer Med 2020 Aug,9(16):5708-18. doi: 10.1002/cam4.3225. Epub 2020 Jun 26. PMID: 32588982; PMCID: PMC7433838.

23. He Y. Mao M, Shi W. He Z, Zhang L. Wang X. Development and validation of a prognostic nomogram in gastric cancer with hepatitis B virus infection. J Transl Med. 2019 Mar 25;17(1):98. doi: 10.1186/s12967-019-1841-3. PMID: 30909980; PMCID: PMC6434786..

24. Ohashi T. Komatsu S, Ichikawa D. Kosuga T, Okamoto K. Konishi H, Shiozaki A. Fujiwara H, Otsuji E. Tumor Index as a Combined Indicator of Tumor Depth and Size in Gastric Cancer. Anticancer Res 2016 Apr,36(4):1895-900. PMID: 27069177.

25. Nishizawa T. Yahagi N. Long-Term Outcomes of Using Endoscopic Submucosal Dissection to Treat Early Gastric Cancer. Gut Liver. 2018 Mar15;12(2):119-124. doi: 10.5009/gnl17095. PMID: 28673068; PMCID: PMC5832335.

26. Nomura E. Okajima K. Function-preserving gastrectomy for gastric cancer in Japan. World J Gastroenterol. 2016 Jul 14,22(26):5888-95. doi: 10.3748/wjg.v22.i26.5888. PMID: 27468183; PMCID: PMC4948261..

27. Li H. Wang C, Wei Z. Chen W, Guo Z. He Y, Zhang C. Differences in the prognosis of gastric cancer patients of different sexes and races and the molecular mechanisms involved. Int J Oncol. 2019 Nov;55(5):1049-68. doi: 10.3892/ijo.2019.4885. Epub 2019 Sep 26. PMID: 31793655; PMCID: PMC6776187.

28. Riihimäki M. Hemminki A, Sundquist K. Sundquist J, Hemminki K. Metastatic spread in patients with gastric cancer. Oncotarget. 2016 Aug 9;7(32):52307-52316. doi: 10.18632/oncotarget.10740. PMID: 27447571 ; PMCID: PMC5239553.

\section{Tables}

Due to technical limitations, the tables are only available as a download in the supplemental files section.

\section{Figures}




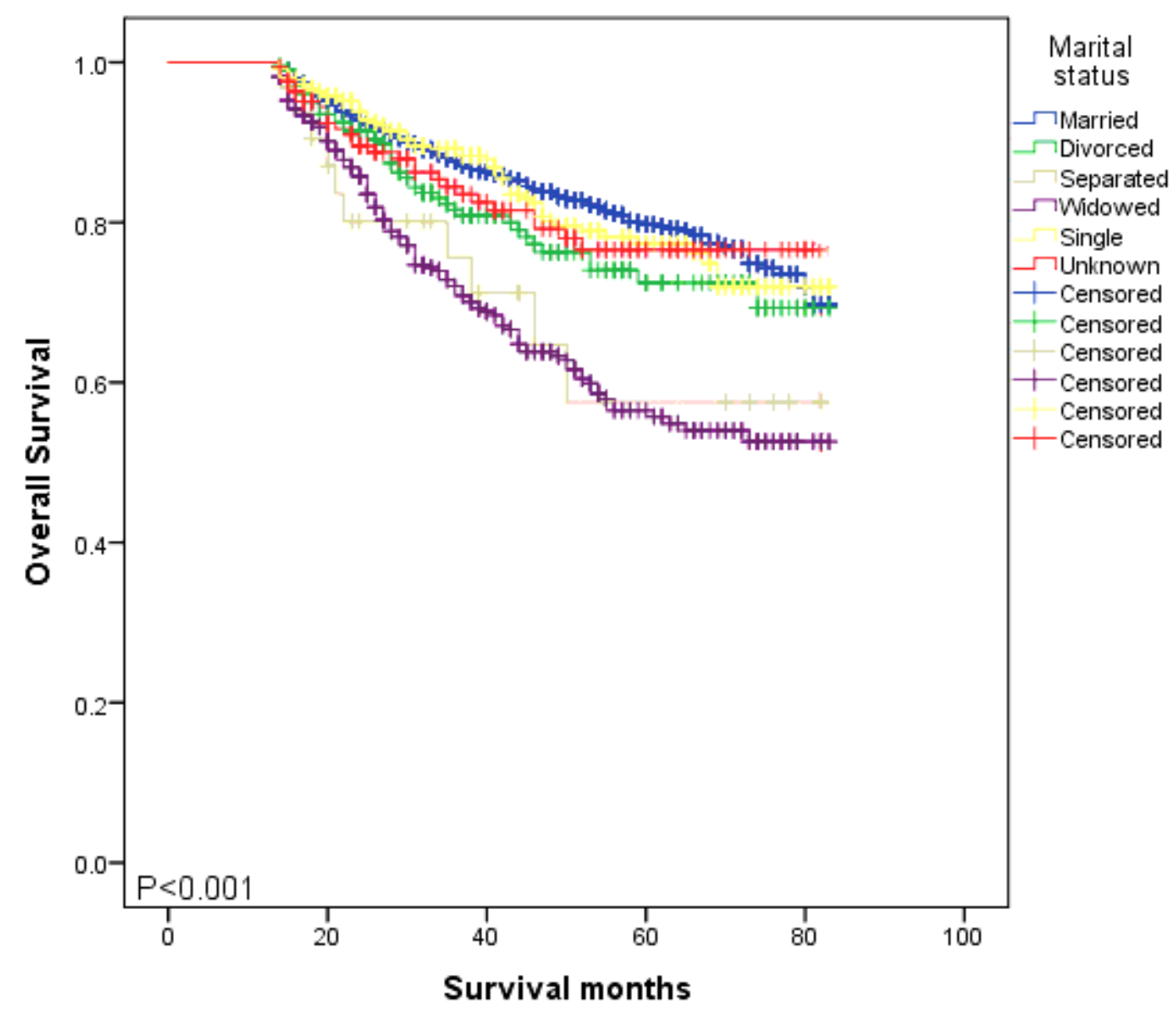

\section{Figure 1}

Kaplan-Meier survival analysis of overall survival among different marital status groups in patients with early-stage gastric cancer $(P<0.001)$.
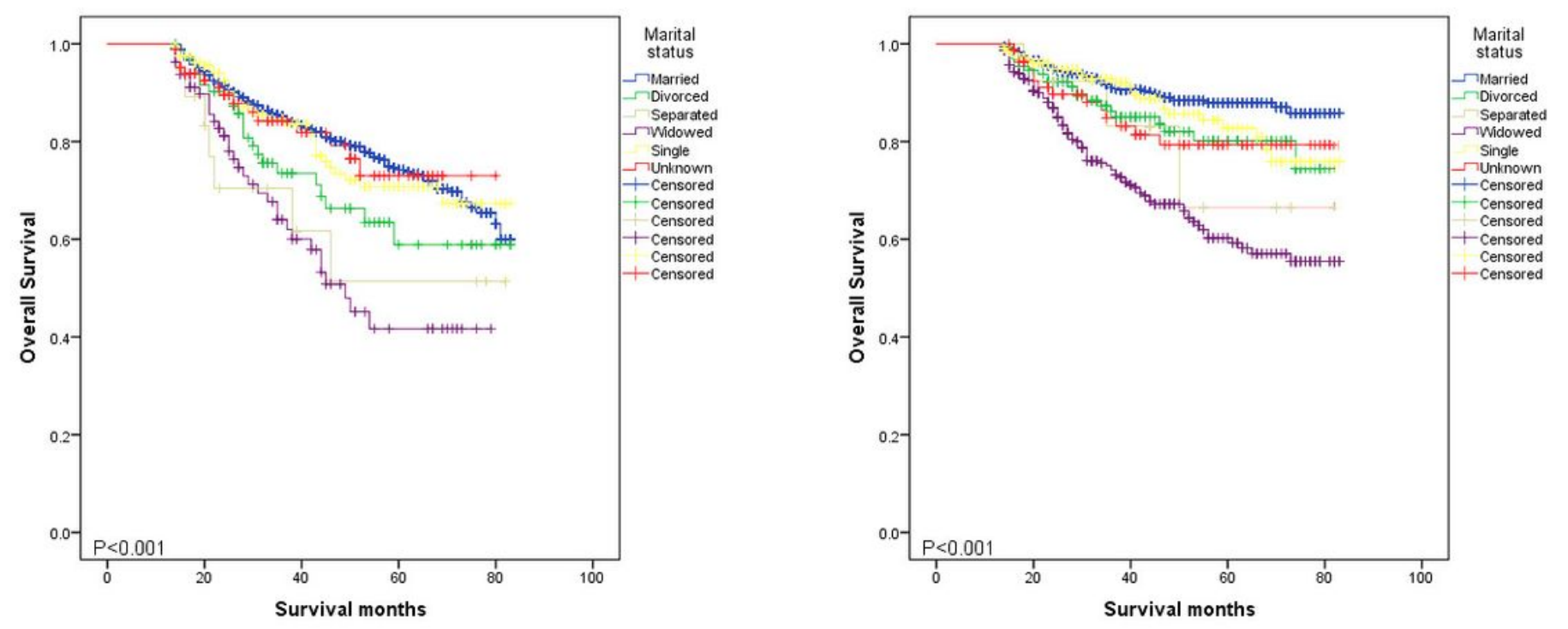

Figure 2 
Kaplan-Meier survival analysis of overall survival among different marital status groups in genders. Overall survival among different marital status groups in (Left panel-A) male patients $(P<0.001)$ and (Right panel-B) female patients $(P<0.001)$ with early-stage gastric cancer.
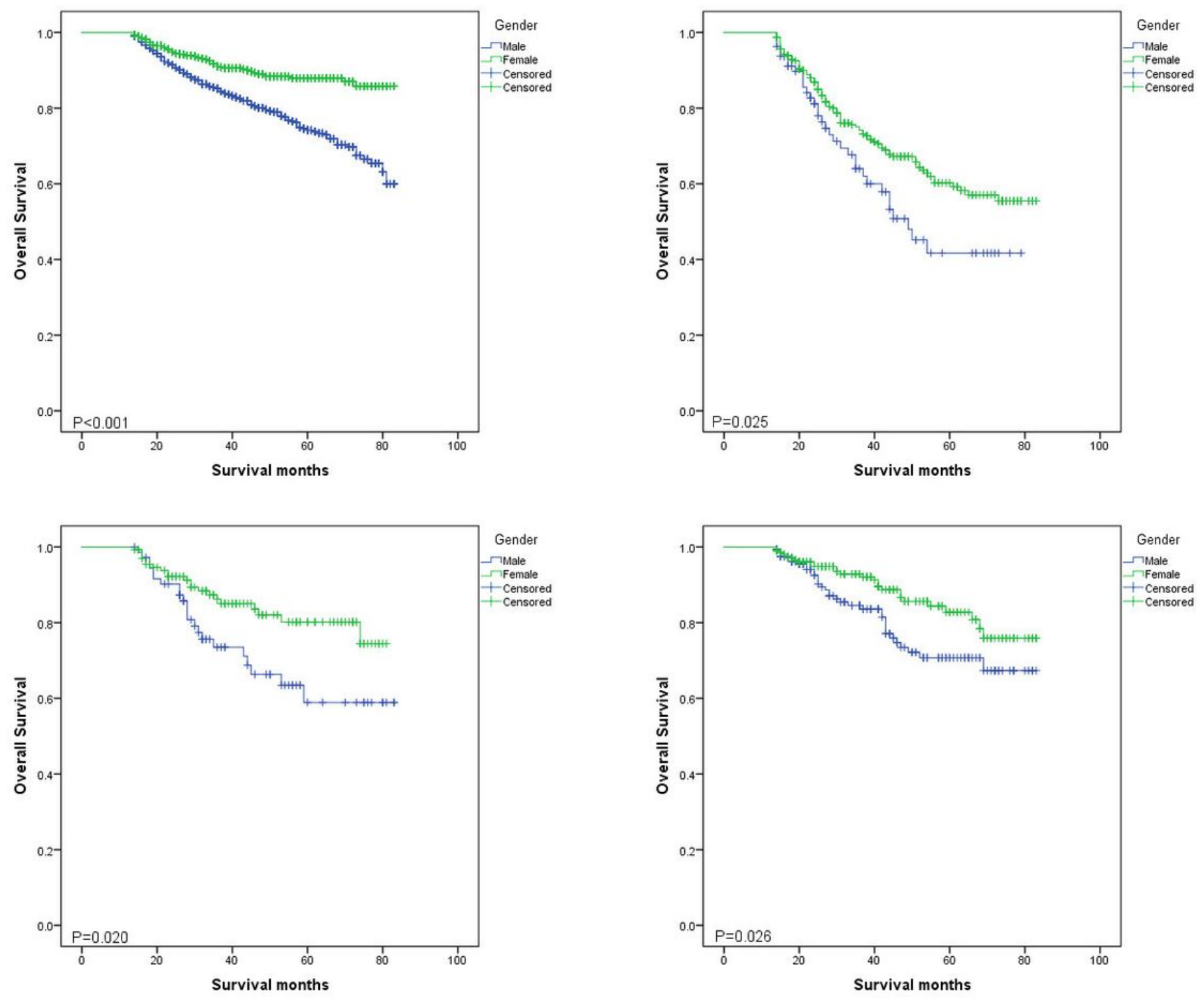

\section{Figure 3}

Kaplan-Meier survival analysis of each known marital status group among different genders, except for the "separated" group. Overall survival (Top left panel-A) between married male and female patients $(P<0.001)$, (Bottom left panel-B) between divorced male and female patients $(P=0.020)$, (Top right panelC) between widowed male and female patients $(P=0.025)$, and (Bottom right panel- $D)$ between single male and female patients $(P=0.026)$. 


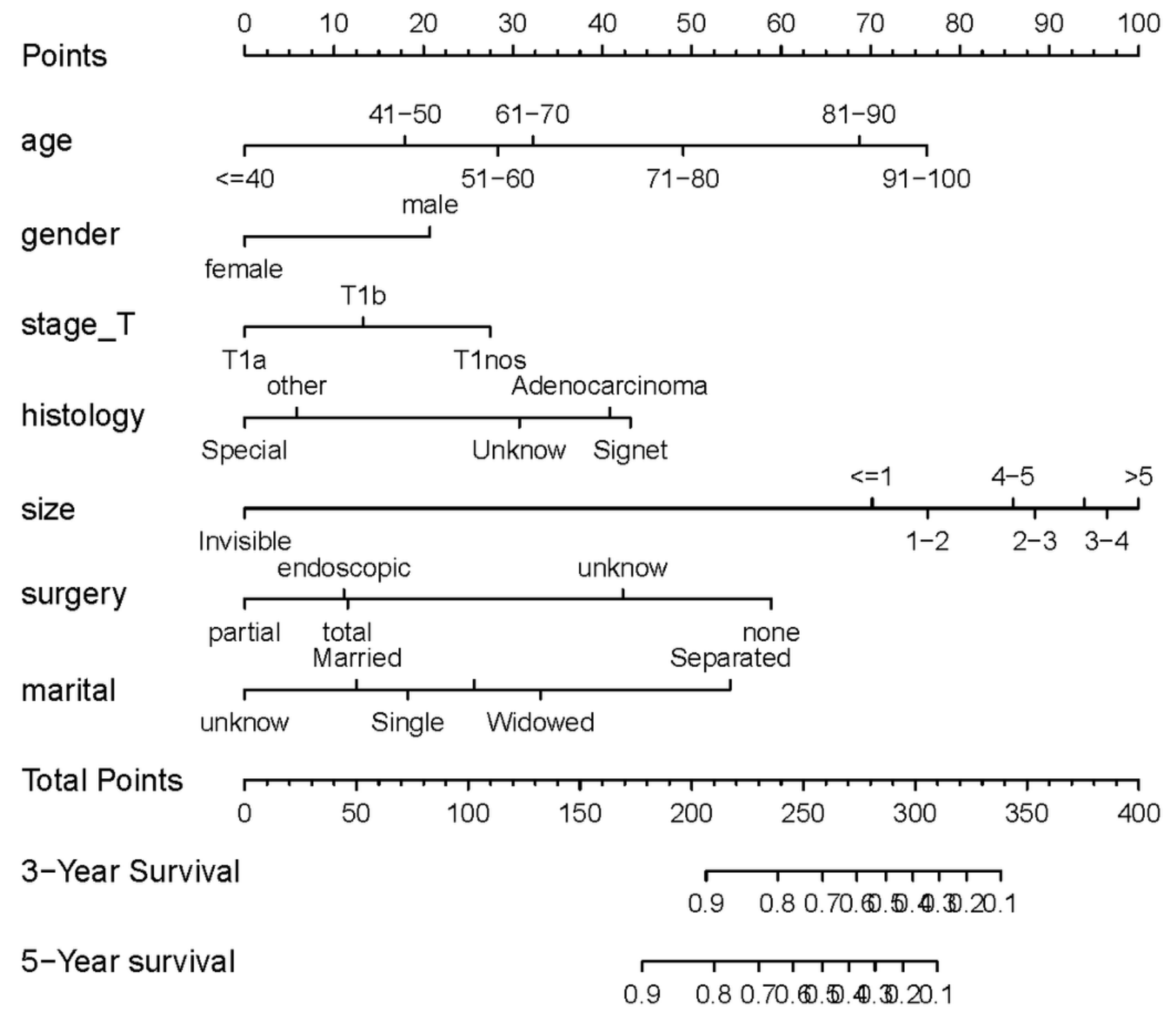

\section{Figure 4}

Nomogram predicting the overall survival of patients with early-stage gastric cancer. 

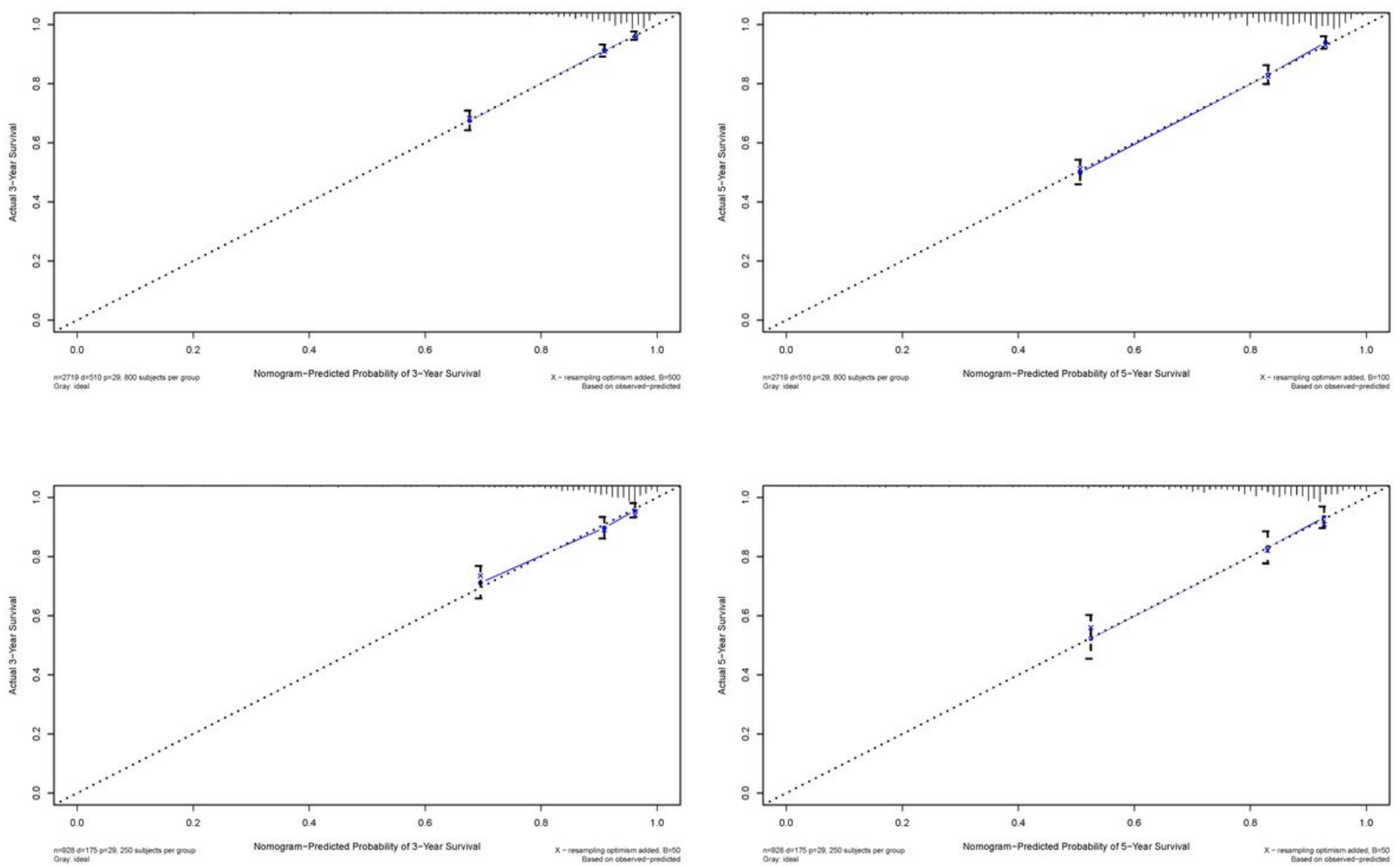

\section{Figure 5}

Calibration curves of the prognostic nomogram for predicting 3- and 5-year overall survivals of patients with early-stage gastric cancer. Three-year overall survival of (Top left panel-A) training set and (Bottom left panel-B) testing set; five-year overall survival of (Top right panel-C) training set and (Bottom right panel-D) testing set 
3-year Survival AUC $=0.774$

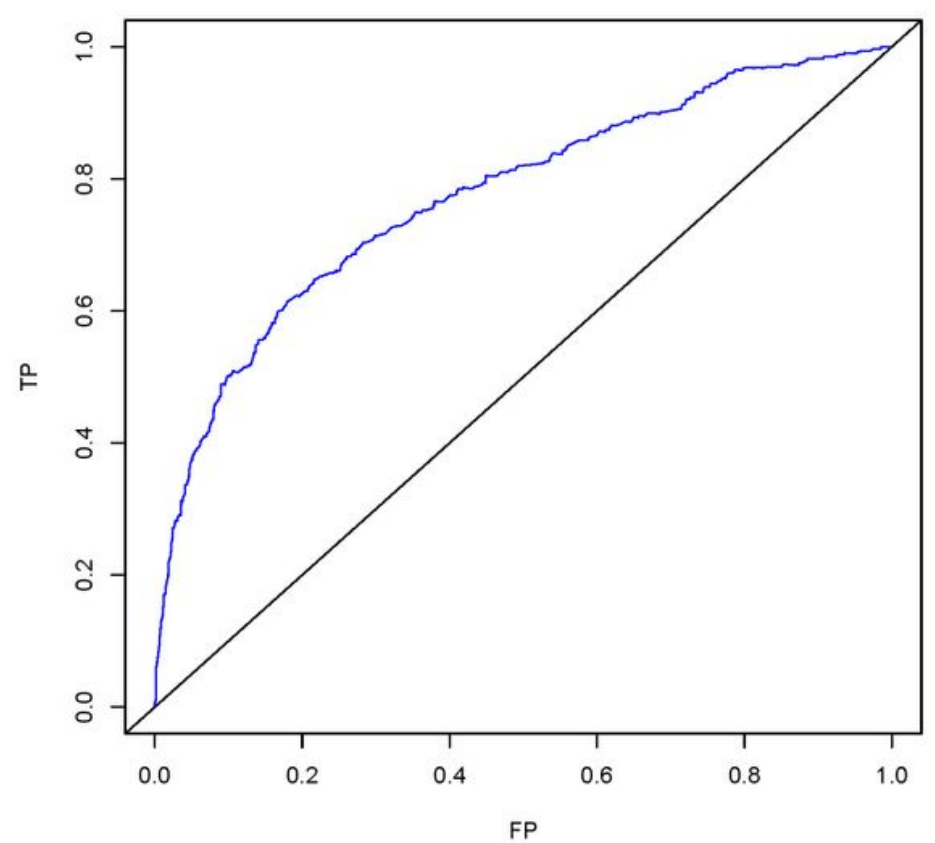

3-year Survival AUC $=0.717$

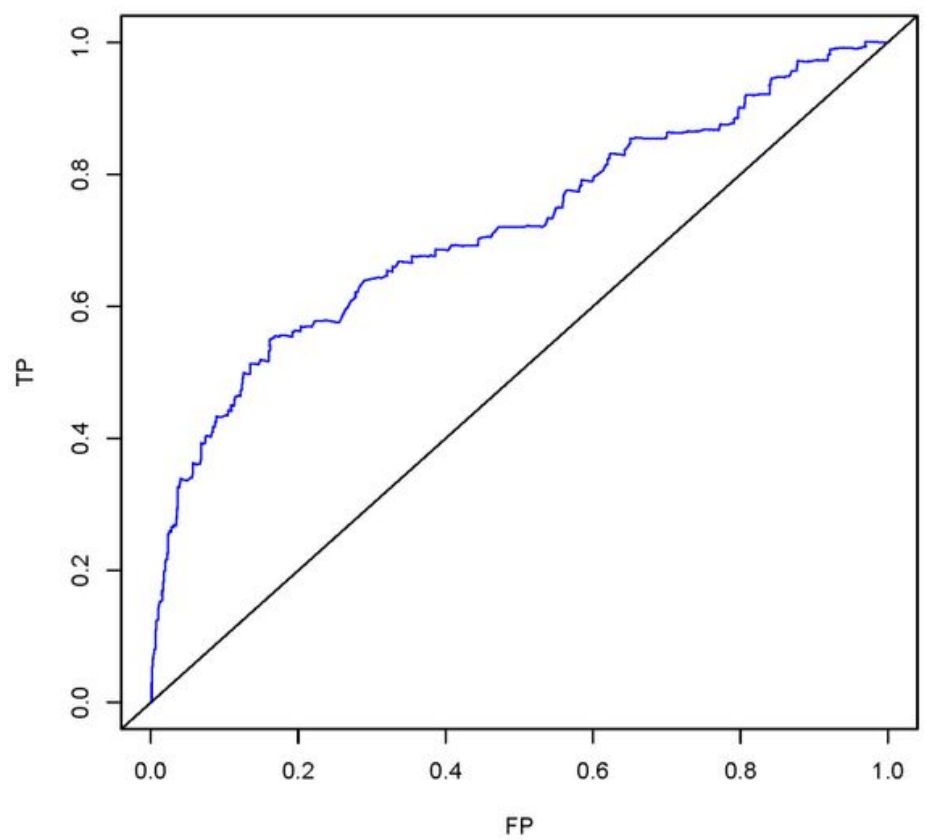

5-year Survival AUC $=0.77321$

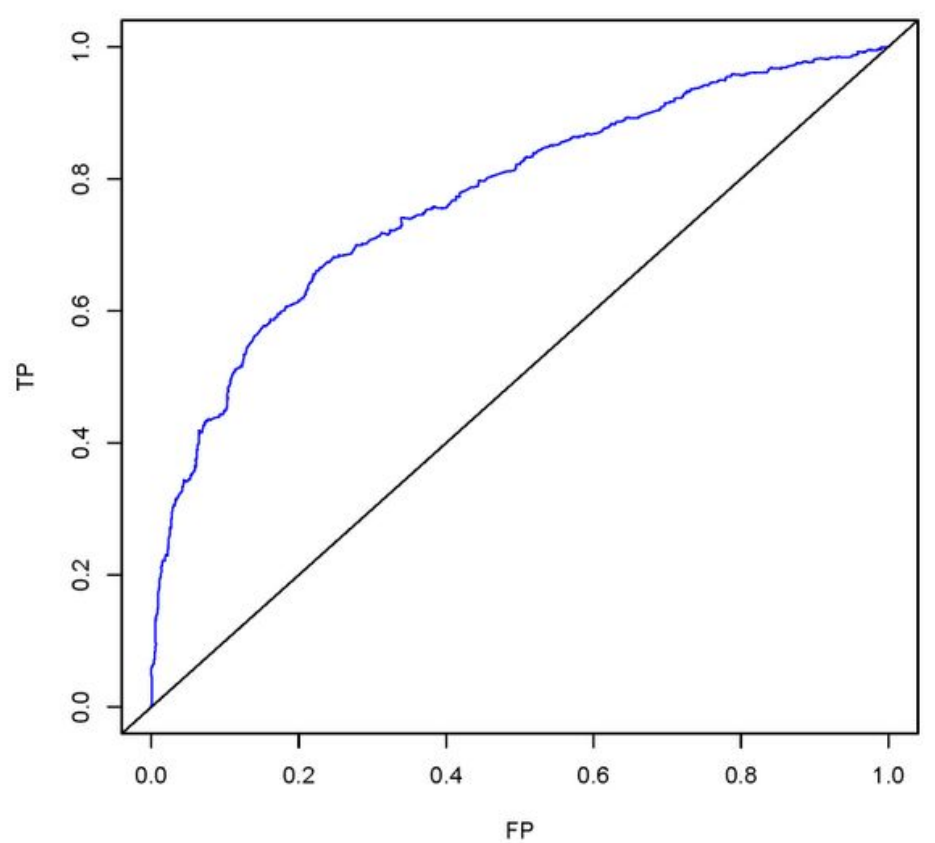

5-year Survival AUC $=0.7221$

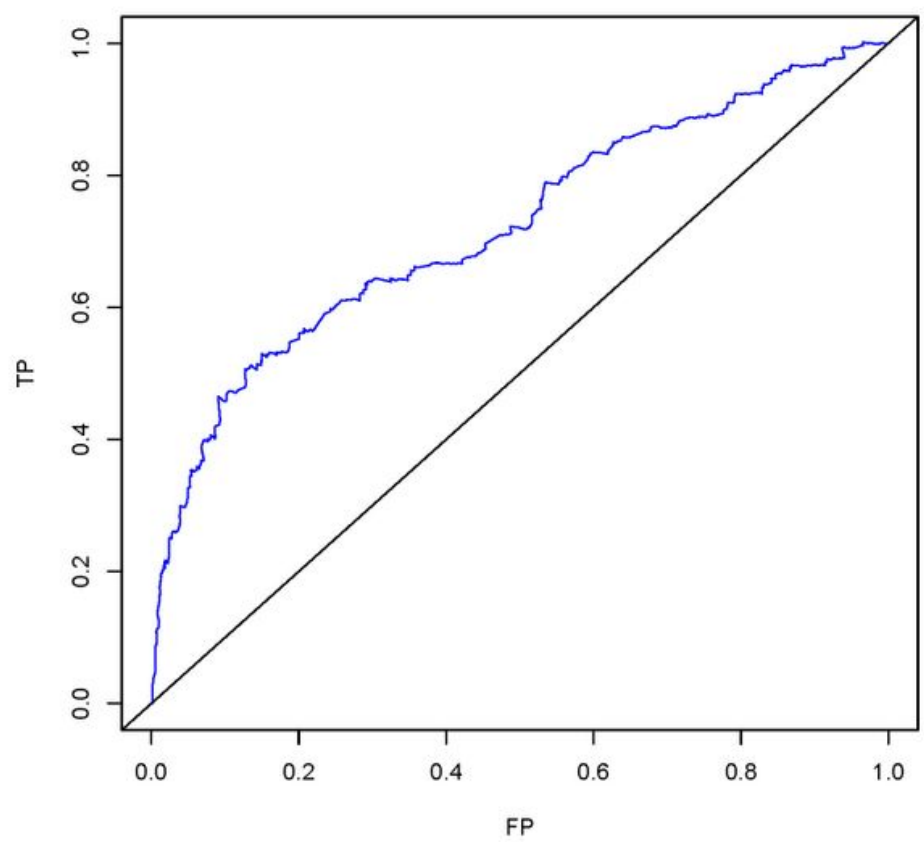

\section{Figure 6}

Receiver operating characteristic curves of 3-year and 5-year survivals. Receiver operating characteristic curves of 3-year survival of (Top left panel-A) training set and (Bottom left panel-B) testing set; Receiver operating characteristic curves of 5-year survival of (Top right panel-C) training set and (Bottom right panel-D) testing set. 


\section{Surival curve of risk score $(\mathrm{P}=0)$}

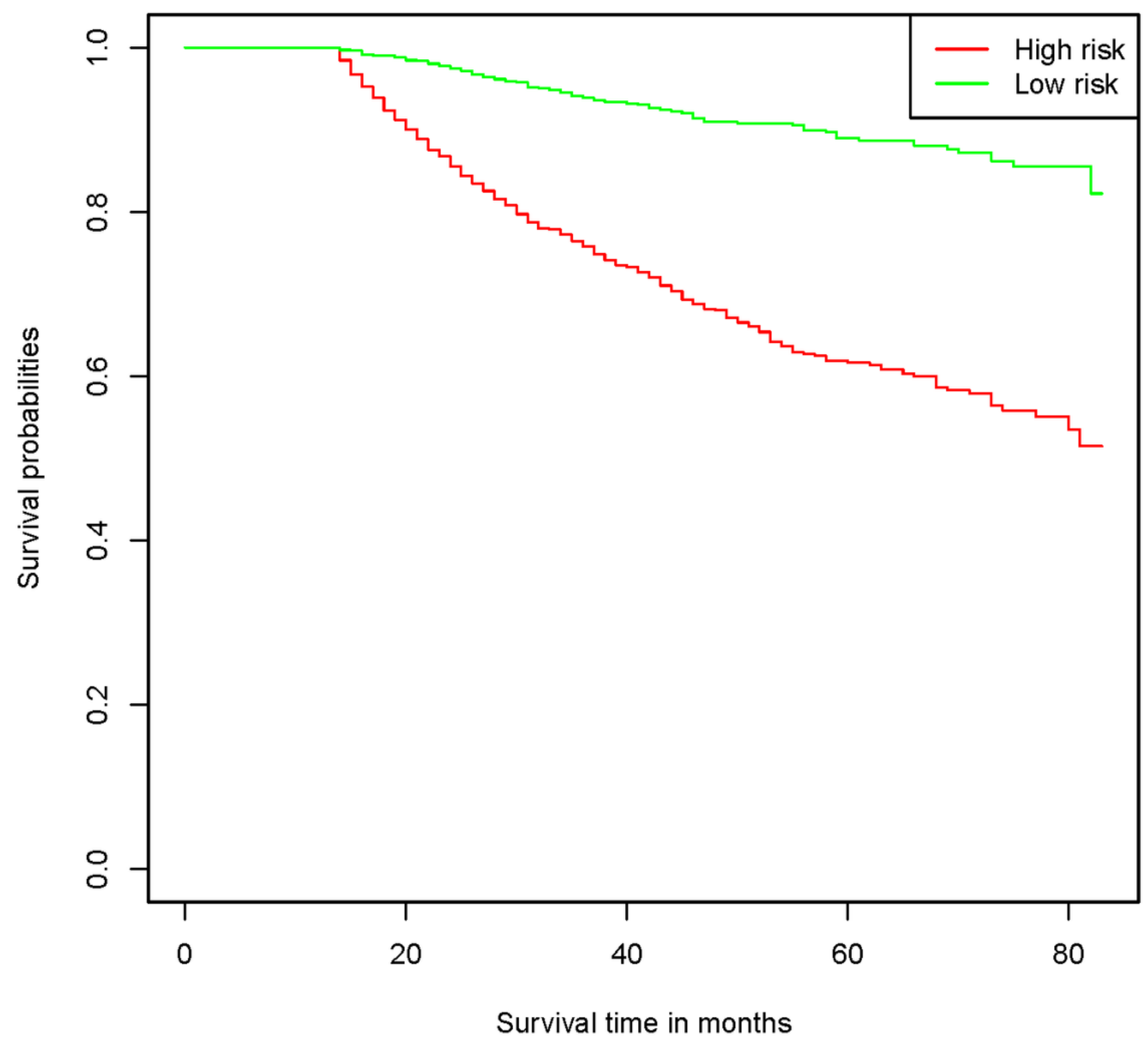

Figure 7

Receiver operating characteristic curves of 3-year and 5-year survivals. Receiver operating characteristic curves of 3-year survival of (A) training set and (B) testing set; Receiver operating characteristic curves of 5 -year survival of (C) training set and (D) testing set.

\section{Supplementary Files}


This is a list of supplementary files associated with this preprint. Click to download.

- Table11.xlsx

- Table21.xIsx

- Table31.xlsx 